\title{
A Petrov-Galerkin reduced basis approximation of the Stokes equation in parametrized geometries
}

\author{
Assyr Abdulle ${ }^{a}$, Ondrej Budáč ${ }^{a}$ \\ a ANMC, Section de Mathématiques, École Polytechnique Fédérale de Lausanne, Switzerland
}

\begin{abstract}
We present a Petrov-Galerkin reduced basis (RB) approximation for the parametrized Stokes equation. Our method, which relies on a fixed solution space and a parameter-dependent test space, is shown to be stable (in the sense of Babuška) and algebraically stable (a bound on the condition number of the online system can be established). Compared to other stable RB methods that can also be shown to be algebraically stable, our approach has a significantly smaller online time cost and general applicability to linear non-coercive problems without assuming a saddle-point structure.
\end{abstract}

\section{Résumé}

Une méthode d'éléments finis de type Petrov-Galerkin pour l'approximation en base réduite du problème de Stokes. Nous présentons une méthode d'éléments finis de type Petrov-Galerkin pour l'approximation en "bases réduites" du problème de Stokes. La stability de notre méthode est établie à l'aide de la théorie inf-sup de Babuška et nous prouvons une borne sur la condition numérique de la matrice du système linéaire "en ligne". Comparée aux méthodes de types bases réduites existantes qui sont à la fois stable et dont la condition numérique du système linéaire en ligne peut être controlée, notre méthode a un coût en ligne considerablement plus faible et est applicable à des formulations générales non-coercives ne nécessitant pas de structure de type point-selle.

\section{Version française abrégée}

Les équations aux dérivées partielles qui dépendent de paramètres sont utilisées dans beaucoup d'applications. Dans cette Note, nous nous intéressons à l'équation de Stokes posée dans un domaine dont la géométrie dépend d'un ou de plusieurs paramètres. L'idée est alors de sélectionner (par exemple par un algorithme glouton) des paramètres représentatifs de la géométrie et de résoudre l'équation de Stokes de façon précise pour ce jeux de paramètres. Ces calculs sont effectués dans une étape "hors ligne". La

Email addresses: assyr.abdulle@epfl.ch (Assyr Abdulle), ondrej.budac@epfl.ch (Ondrej Budáč).

URL: http://anmc.epfl.ch/abdulle.html (Assyr Abdulle). 
résolution de l'équation pour de nouveaux paramètres lors d'une application particulière (phase "en ligne") sera alors obtenue de façon beaucoup moins couteuse en "interpolant" la base de solutions préalablement calculées (pour plus de détails voir par exemple [2,5]).

Pour que de telles méthodes soient efficace il est important que le système linéaire en ligne soit de dimension aussi petite que possible tout en étant bien conditionné (algébriquement stable). Or pour les méthodes les plus efficaces rapportées dans la littérature (voir [5, Paragraphe 4]), la stabilité ne peut pas être établie.

Dans cette Note, nous proposons une méthode de base réduite pour le problème de Stokes dont la stabilité (au sens d'une condition inf-sup) et la stabilité algébrique peuvent être établies et dont la taille du système linéaire en ligne est comparable aux méthodes les plus efficaces [5]. De plus, notre méthode est applicable à des formulations générales non-coercives ne nécessitant pas de structure de type point-selle.

\section{Introduction}

Consider a weak formulation of a differential equation and an output of interest (function of the solution) that depend on a parameter. For any allowed parameter value, one can numerically approximate the solution (e.g., using finite elements) and then compute an approximation of the output. A repeated evaluation of the output of interest for different parameters (needed for example in optimization) can become prohibitively time-consuming. Reduced basis (RB) methods can be applied to speed up this evaluation by projecting the problem to a low-dimensional RB space (see [6] for a general review). This $\mathrm{RB}$ space is spanned by solutions (or other related functions) computed for a sample set of parameter points. Efficiency is achieved via splitting of the computation into two parts. In the offline part, performed only once, the RB space is constructed. In the online part, which can be performed repeatedly and for any parameter value, precomputed values from the offline part are used for a fast evaluation of the output of interest and a posteriori error estimates.

In this paper we are interested in the parametrized Stokes problems solved by the RB method. Efficiency and reliability of the RB approximation depend on two stability properties of the reduction: the approximation stability (inf-sup condition in the reduced problem) and the algebraic stability (bounded condition number of the reduced linear system). For a recent review of the RB method for the Stokes equation we refer to $[2,5]$, where it is shown how a saddle-point structure can be exploited to derive RB methods for the Stokes equation. The common principle of these methods is to build the RB space with separate velocity and pressure samples and then enrich the velocity space to achieve the inf-sup stability of the reduction in the sense of Brezzi. These methods are mentioned in greater detail at the end of Section 2.

In this paper we treat the Stokes equation as a non-coercive problem with no structural assumptions (as a saddle-point structure). Such an approach is indeed useful, e.g., when we have a nested saddlepoint structure, where additional Lagrange multipliers are used to enforce boundary conditions [1]. One can then completely abandon the Brezzi stability theory and concentrate solely on the inf-sup condition in the sense of Babuška. We present an a priori stable RB method for the Stokes equation that uses a Petrov-Galerkin projection with a fixed solution space and a parameter-dependent test space. This approach has several advantages:

- a priori (approximation and algebraic) stability,

- size of the online linear system equal to the number of selected parameter samples,

- simple description (allowing black-box usage) that doesn't assume any structure (e.g., saddle-point). We note that RB methods for parametrized linear non-coercive problems have been proposed in [4] but have not yet been derived for the Stokes equation. 
The article is structured as follows. In Section 2 we describe the transformation of the Stokes equation from a parametrized domain to a reference domain. Section 3 presents the mentioned RB method for the Stokes equation and we conclude by a numerical experiment in Section 4.

\section{Parametrized Stokes equation}

Let $d \in\{2,3\}$ and $\Omega \subset \mathbb{R}^{d}$ be an open connected domain with Lipschitz boundary $\partial \Omega$ divided into a Dirichlet part $\Gamma_{\mathrm{D}}$ and a Neumann part $\Gamma_{\mathrm{N}}$. Assume that both $\Gamma_{\mathrm{D}}$ and $\Gamma_{\mathrm{N}}$ are non-empty and define a velocity space $V=\left\{\mathbf{v} \in H^{1}(\Omega)^{d}:\left.\mathbf{v}\right|_{\Gamma_{\mathrm{D}}} \equiv 0\right\}$ and a pressure space $Q=L^{2}(\Omega)$. For the sake of simplicity assume that a constant forcing term $\mathbf{f} \in \mathbb{R}^{d}$ is given and consider a weak formulation of the well-posed homogeneous Stokes equation in $\Omega$. Find $\mathbf{u} \in V$ and $p \in Q$ such that

$$
\begin{aligned}
& \int_{\Omega} \nabla \mathbf{u}: \nabla \mathbf{v} \mathrm{d} x-\int_{\Omega} p(\operatorname{div} \mathbf{v}) \mathrm{d} x \quad=\int_{\Omega} \mathbf{f} \cdot \mathbf{v} \quad \forall \mathbf{v} \in V, \\
& -\int_{\Omega} q(\operatorname{div} \mathbf{u}) \mathrm{d} x \quad=0 \quad \forall q \in Q,
\end{aligned}
$$

where $\nabla \mathbf{u}: \nabla \mathbf{v}=\sum_{i=1}^{n} \nabla \mathbf{u}_{i} \cdot \nabla \mathbf{v}_{i}$.

Let us call $\Omega$ the reference domain and consider a class of Stokes problems in domains $\Omega^{\mu} \subset \mathbb{R}^{d}$ (with $\left.\partial \Omega^{\mu}=\Gamma_{\mathrm{D}}^{\mu} \cup \Gamma_{\mathrm{N}}^{\mu}\right)$ parametrized by $\mu \in \mathcal{D}$, where $\mathcal{D} \subset \mathbb{R}^{P}$ is a parametric space and $P \in \mathbb{N}$. Instead of studying (1) in a different domain $\Omega^{\mu}$ for every $\mu \in \mathcal{D}$, we map the Stokes equation from $\Omega^{\mu}$ to $\Omega$ via change of variables. Assume that a mapping $\varphi: \bar{\Omega} \times \mathcal{D} \rightarrow \mathbb{R}^{d}$ is known such that for every $\mu \in \mathcal{D}$ the $\operatorname{map} \varphi(\cdot ; \mu): \bar{\Omega} \rightarrow \bar{\Omega}^{\mu}$ is a homeomorphism with $\varphi\left(\Gamma_{\mathrm{D}} ; \mu\right)=\Gamma_{\mathrm{D}}^{\mu}$. Moreover, let $R \in \mathbb{N}$ and assume that $\left\{\Omega_{r}\right\}_{r=1}^{R}$ is a disjoint decomposition of $\Omega$ such that for $r \in\{1, \ldots, R\}$ the map $\left.\varphi(\cdot ; \mu)\right|_{\Omega_{r}}$ is affine, that is, there are $C^{r}: \mathcal{D} \rightarrow \mathbb{R}^{d}$ and $G^{r}: \mathcal{D} \rightarrow \mathbb{R}^{d \times d}$ such that $\left.\varphi(x ; \mu)\right|_{\Omega_{r}}=G^{r}(\mu) x+C^{r}(\mu)$. Taking (1) in the domain $\Omega^{\mu}$ and using the change of variables $x_{\text {new }}=\varphi\left(x_{\mathrm{old}} ; \mu\right)^{-1}$ we obtain the following formulation: find $\mathbf{u}(\mu) \in V$ and $p(\mu) \in Q$ such that

$$
\begin{aligned}
& a(\mathbf{u}(\mu), \mathbf{v} ; \mu)+b(\mathbf{v}, p(\mu) ; \mu)=F(\mathbf{v} ; \mu) \quad \forall \mathbf{v} \in V, \\
& b(\mathbf{u}(\mu), q ; \mu) \quad=0 \quad \forall q \in Q,
\end{aligned}
$$

where the parametric bilinear forms $a: V \times V \times \mathcal{D} \rightarrow \mathbb{R}$ and $b: V \times Q \times \mathcal{D} \rightarrow \mathbb{R}$ and the parametric linear form $F: V \times \mathcal{D} \rightarrow \mathbb{R}$ can be expressed as

$$
\begin{aligned}
a(\mathbf{w}, \mathbf{v} ; \mu) & =\sum_{r=1}^{R} \sum_{i, j=1}^{d} \nu_{i j}^{r}(\mu) \int_{\Omega_{r}} \frac{\partial \mathbf{w}}{\partial x_{i}} \cdot \frac{\partial \mathbf{v}}{\partial x_{j}} \mathrm{~d} x, & & \nu^{r}(\mu)=\operatorname{det}\left(G^{r}(\mu)\right) G^{r}(\mu)^{-1} G^{r}(\mu)^{-T}, \\
b(\mathbf{v}, q ; \mu) & =-\sum_{r=1}^{R} \sum_{i, j=1}^{d} \theta_{i j}^{r}(\mu) \int_{\Omega_{r}} q \frac{\partial \mathbf{v}_{i}}{\partial x_{j}} \mathrm{~d} x, & \theta^{r}(\mu) & =\operatorname{det}\left(G^{r}(\mu)\right) G^{r}(\mu)^{-T}, \\
F(\mathbf{v} ; \mu) & =\sum_{r=1}^{R} \Theta_{r}^{F}(\mu) \int_{\Omega_{r}} \mathbf{f} \cdot \mathbf{v} \mathrm{d} x, & \Theta_{r}^{F}(\mu) & =\operatorname{det}\left(G^{r}(\mu)\right) .
\end{aligned}
$$

The problem (2) together with the affine decomposition (3) are instrumental to the RB methods for Stokes problems. Standard RB methods for (2), as documented in [2,5], proceed as follows. Given $N \in \mathbb{N}$ parameter sample points $\mu^{1}, \ldots, \mu^{N} \in \mathcal{D}$, the pressure RB space is set to $\operatorname{span}\left\{p\left(\mu^{n}\right), 1 \leq n \leq N\right\}$. The velocity $\mathrm{RB}$ space includes $\operatorname{span}\left\{\mathbf{u}\left(\mu^{n}\right), 1 \leq n \leq N\right\}$ but it is further enriched to achieve good approximation and stability. We mention two exemplary ways for this enrichment: 
(i) adding $N$ more basis functions to the velocity RB space by taking the so-called pressure supremizers, evaluated at the parameter values of the pressure samples. An online linear system of size $3 N$ is obtained without a priori guarantee of its stability.

(ii) adding $N Q_{b}$ more basis functions to the velocity $\mathrm{RB}$ space by taking the so-called partial pressure supremizers, where $Q_{b}$ is the number of terms in an affine decomposition of $b(\cdot, \cdot ; \mu)$. Here one can show approximation and algebraic stability but the online time cost can become very expensive, since a dense linear system of size $\left(Q_{b}+2\right) N$ needs to be solved.

In the following section we describe a RB method for the Stokes equation that has online time cost very close to the fast method (i) and a priori stability properties as the method (ii).

\section{A Petrov-Galerking RB method for the Stokes equation}

Let $X=V \times Q$ and denote by $(\cdot, \cdot)_{X}$ a scalar product on $X$ and let $\|\cdot\|_{X}$ be the corresponding norm. Consider a conformal simplicial mesh of $\Omega$ and a stable pair of velocity and pressure finite elements, e.g., the $\mathbb{P}_{2} / \mathbb{P}_{1}$ Taylor-Hood elements. Let $\mathcal{N} \in \mathbb{N}$ be the number of degrees of freedom of this discretization and denote the (product) finite element space by $X^{\mathcal{N}} \subset X$. We write an approximation to (2) as a single symmetric non-coercive problem: find $\mathbf{U}(\mu) \in X^{\mathcal{N}}$ such that

$$
A(\mathbf{U}(\mu), \mathbf{V} ; \mu)=F(\mathbf{V} ; \mu) \quad \forall \mathbf{V} \in X^{\mathcal{N}}
$$

where the bilinear form $A(\cdot, \cdot ; \mu): X \times X \rightarrow \mathbb{R}$ is defined by $A((\mathbf{u}, p),(\mathbf{v}, q) ; \mu)=a(\mathbf{u}, \mathbf{v} ; \mu)+b(\mathbf{v}, p ; \mu)+$ $b(\mathbf{u}, q ; \mu)$. The form $A(\cdot, \cdot ; \mu)$ and the forcing term $F(\cdot ; \mu): X \rightarrow \mathbb{R}$ are affine in the parameter. Indeed, we have $A(\mathbf{W}, \mathbf{V} ; \mu)=\sum_{q=1}^{Q_{A}} \Theta_{q}^{A}(\mu) A^{q}(\mathbf{W}, \mathbf{V})$, where (3) ensures the existence bilinear forms $A^{q}: X \times$ $X \rightarrow \mathbb{R}$ and functions $\Theta_{q}^{A}: \mathcal{D} \rightarrow \mathbb{R}$ for $q \in\left\{1, \ldots, Q_{A}\right\}$, where $Q_{A} \leq 3 R d^{2}$. Similarly, $F(\mathbf{V} ; \mu)=$ $\sum_{q=1}^{R} \Theta_{q}^{F}(\mu) F^{q}(\mathbf{V})$, where functionals $F^{q}: X \rightarrow \mathbb{R}$ are easily deductible from (3).

Notice that (4) does not assume any special structure (as saddle-point). We assume that (4) is uniformly continuous, i.e., there exists $\gamma_{\max } \in \mathbb{R}$ such that $\gamma(\mu)=\|A(\cdot, \cdot ; \mu)\| \leq \gamma_{\max }$ for every $\mu \in \mathcal{D}$ and that the following uniform inf-sup condition holds: there is $\beta_{\min }>0$ such that

$$
\beta_{\mathcal{N}}(\mu)=\inf _{\mathbf{W} \in X^{\mathcal{N}}} \sup _{\mathbf{V} \in X^{\mathcal{N}}} \frac{A(\mathbf{W}, \mathbf{V} ; \mu)}{\|\mathbf{W}\|_{X}\|\mathbf{V}\|_{X}} \geq \beta_{\min } \quad \forall \mu \in \mathcal{D} .
$$

We next describe the RB spaces and a low-dimensional projection of $(4)$. Let $\mu^{1}, \ldots, \mu^{N} \in \mathcal{D}$ be $N \in \mathbb{N}$ sample points and let

$$
X^{N}=\operatorname{span}\left\{\mathbf{U}\left(\mu^{n}\right), 1 \leq n \leq N\right\}
$$

be the solution RB space. Stability of the RB approximation is achieved by using a Petrov-Galerking projection, where the test space is selected as an image of the so-called supremizer operator $T^{\mu}: X^{\mathcal{N}} \rightarrow$ $X^{\mathcal{N}}$ applied on $X^{N}$. For any $\mathbf{W} \in X^{\mathcal{N}}$ let $T^{\mu}(\mathbf{W}) \in X^{\mathcal{N}}$ be such that $\left(T^{\mu}(\mathbf{W}), \mathbf{V}\right)_{X}=A(\mathbf{W}, \mathbf{V} ; \mu)$ for every $\mathbf{V} \in X^{\mathcal{N}}$. Define the parameter-dependent test space

$$
X^{N, \mu}=\operatorname{span}\left\{T^{\mu}\left(\mathbf{U}\left(\mu^{n}\right)\right), 1 \leq n \leq N\right\} .
$$

The RB projection of (4) then reads: find $\mathbf{U}^{N}(\mu) \in X^{N}$ such that

$$
A\left(\mathbf{U}^{N}(\mu), \mathbf{V} ; \mu\right)=F(\mathbf{V} ; \mu) \quad \forall \mathbf{V} \in X^{N, \mu} .
$$

Lemma 3.1 (approximation stability) The formulation (5) preserves the inf-sup stability, that is,

$$
\beta_{N}(\mu)=\inf _{\mathbf{W} \in X^{N}} \sup _{\mathbf{V} \in X^{N, \mu}} \frac{A(\mathbf{W}, \mathbf{V} ; \mu)}{\|\mathbf{W}\|_{X}\|\mathbf{V}\|_{X}} \geq \beta_{\mathcal{N}}(\mu) .
$$


The proof is elementary. Using $X^{N}=T^{\mu}\left(X^{N}\right)$ and $A(\mathbf{W}, \mathbf{V} ; \mu)=\left(T^{\mu}(\mathbf{W}), \mathbf{V}\right)_{X} \leq\left\|T^{\mu}(\mathbf{W})\right\|_{X}\|\mathbf{V}\|_{X}$, where equality occurs for $\mathbf{V}=T^{\mu}(\mathbf{W})$, we get

$$
\beta_{N}(\mu)=\inf _{\mathbf{W} \in X^{N}} \frac{\left\|T^{\mu}(\mathbf{W})\right\|_{X}}{\|\mathbf{W}\|_{X}} \geq \inf _{\mathbf{W} \in X^{\mathcal{N}}} \frac{\left\|T^{\mu}(\mathbf{W})\right\|_{X}}{\|\mathbf{W}\|_{X}}=\beta_{\mathcal{N}}(\mu) .
$$

Having defined the RB problem (5), we are left with a description of the offline-online decoupling, where the goal is to obtain an online time cost independent of $\mathcal{N}$. This is rather technical but also standard, the main difficulty being how to deal with the parameter-dependent test spaces.

Let $\boldsymbol{\zeta}_{1}, \ldots, \boldsymbol{\zeta}_{N}$ be an orthonormal basis of $X^{N}$. Notice that $T^{\mu}(\mathbf{W})=\sum_{q=1}^{Q_{A}} T^{q}(\mathbf{W})$, where $T^{q}: X^{\mathcal{N}} \rightarrow$ $X^{\mathcal{N}}$ is a linear operator defined as a solution to the problem: find $T^{q}(\mathbf{W}) \in X^{\mathcal{N}}$ such that $\left(T^{q}(\mathbf{W}), \mathbf{V}\right)_{X}=$ $A^{q}(\mathbf{W}, \mathbf{V})$ for every $\mathbf{V} \in X^{\mathcal{N}}$. Hence, the functions $T^{\mu}\left(\boldsymbol{\zeta}_{n}\right)=\sum_{q=1}^{Q_{A}} \Theta_{q}^{A}(\mu) T^{q}\left(\boldsymbol{\zeta}_{n}\right)$ for $1 \leq n \leq N$ span $X^{N, \mu}$. Writing the RB solution as a linear combination of basis functions $\mathbf{U}^{N}(\mu)=\sum_{n=1}^{N} \bar{U}_{n}(\mu) \boldsymbol{\zeta}_{n}$ and similarly expressing the test functions, we obtain the online reduced system $\bar{A}^{N}(\mu) \bar{U}(\mu)=\bar{F}^{N}(\mu)$ with the matrix $\bar{A}^{N}(\mu) \in \mathbb{R}^{N \times N}$ and the right-hand side $\bar{F}^{N}(\mu) \in \mathbb{R}^{N}$ given by

$$
\bar{A}_{n m}^{N}(\mu)=\sum_{q, r=1}^{Q_{A}} \Theta_{q}^{A}(\mu) \Theta_{r}^{A}(\mu) \underline{A^{q}\left(\boldsymbol{\zeta}_{n}, T^{r}\left(\boldsymbol{\zeta}_{m}\right)\right)}, \quad \bar{F}_{n}^{N}(\mu)=\sum_{q=1}^{Q_{A}} \sum_{r=1}^{R} \Theta_{q}^{A}(\mu) \Theta_{r}^{F}(\mu) \underline{F^{r}\left(T^{q}\left(\boldsymbol{\zeta}_{n}\right)\right)} .
$$

The underlined quantities in (6) can be precomputed in the offline part and are obtained by a Greedy procedure controlled by appropriate a posteriori error estimators mentioned below. Notice that the size of the online linear system (6) is only $N$.

Lemma 3.2 (algebraic stability) The condition number of $\bar{A}^{N}(\mu)$ is at most $\gamma(\mu)^{2} / \beta_{\mathcal{N}}(\mu)^{2}$.

Proof. Using the Cauchy-Schwartz inequality one obtains $\gamma(\mu)\|\mathbf{W}\|_{X} \geq\left\|T^{\mu}(\mathbf{W})\right\|_{X} \geq \beta_{\mathcal{N}}(\mu)\|\mathbf{W}\|_{X}$ for every $\mathbf{W} \in X^{\mathcal{N}}$. Then, using the orthonormality of $\left\{\boldsymbol{\zeta}_{n}\right\}_{n=1}^{N}$, we get $\beta_{\mathcal{N}}(\mu)^{2}|\bar{W}|^{2} \leq \bar{A}^{N}(\mu) \bar{W} \cdot \bar{W} \leq$ $\gamma(\mu)^{2}|\bar{W}|^{2}$ for any $\bar{W} \in \mathbb{R}^{N}$. The symmetry of $\bar{A}^{N}(\mu)$ then concludes the proof.

A posteriori error evaluation and the greedy construction of the RB spaces uses these inequalities:

$$
\left\|\mathbf{U}(\mu)-\mathbf{U}^{N}(\mu)\right\|_{X} \leq \frac{\left\|R_{\mathrm{pr}}^{N}(\cdot ; \mu)\right\|_{\left(X^{\mathcal{N}}\right)^{\prime}}}{\beta_{\mathcal{N}}(\mu)}, \quad\left|F(\mathbf{U}(\mu) ; \mu)-F\left(\mathbf{U}^{N}(\mu) ; \mu\right)\right| \leq \frac{\left\|R_{\mathrm{pr}}^{N}(\cdot ; \mu)\right\|_{\left(X^{\mathcal{N}}\right)^{\prime}}^{2}}{\beta_{\mathcal{N}}(\mu)},
$$

where $R_{\mathrm{pr}}^{N}(\mathbf{W} ; \mu)=F(\mathbf{W} ; \mu)-A\left(\mathbf{W}, \mathbf{U}^{N}(\mu) ; \mu\right)$ is the (primal) residual.

General outputs of Interest. The right inequality in $(7)$ shows that the loading $F(\mathbf{U}(\mu) ; \mu)$, also called the compliant output, can be estimated with improved (quadratic) accuracy. Consider now a general output of interest $S(\mu)=L(\mathbf{U}(\mu) ; \mu)$, where $L: X \times \mathcal{D} \rightarrow \mathbb{R}$ is affine in the parameter. To improve accuracy, we augment the system (4) with the dual problem: find $\boldsymbol{\Psi}(\mu) \in X^{\mathcal{N}}$ such that

$$
A(\mathbf{W}, \Psi(\mu) ; \mu)=-L(\mathbf{W} ; \mu), \quad \forall \mathbf{W} \in X^{\mathcal{N}} .
$$

We independently use the same RB method (Lemma 3.1 and 3.2 are still valid) to obtain a dual RB solution space $X_{\mathrm{du}}^{M}$ and test space $X_{\mathrm{du}}^{M, \mu}$ based on a sample set $\mu_{\mathrm{du}}^{1}, \ldots, \mu_{\mathrm{du}}^{M} \in \mathcal{D}$, constructed in an offline stage similarly as $X^{N}$ and $X^{N, \mu}$. Using the RB solution $\Psi^{M}(\mu) \in X_{\mathrm{du}}^{M}$ we define an output estimate $S^{N \cdot M}(\mu)=L\left(\mathbf{U}^{N}(\mu) ; \mu\right)-R_{\mathrm{pr}}^{N}\left(\Psi^{M}(\mu) ; \mu\right)$, which satisfies

$$
\left|S(\mu)-S^{N, M}(\mu)\right| \leq \beta_{\mathcal{N}}(\mu)^{-1}\left\|R_{\mathrm{pr}}^{N}(\cdot ; \mu)\right\|_{\left(X^{\mathcal{N}}\right)^{\prime}}\left\|R_{\mathrm{du}}^{M}(\cdot ; \mu)\right\|_{\left(X^{\mathcal{N}}\right)^{\prime}},
$$

where the dual residual is $R_{\mathrm{du}}^{M}(\mathbf{W} ; \mu)=-L(\mathbf{W} ; \mu)-A\left(\mathbf{W}, \mathbf{\Psi}^{M}(\mu) ; \mu\right)$. The evaluation of the output of interest and of the error bounds can be also split into an offline and an online part [4]. The inf-sup constants $\beta_{\mathcal{N}}(\mu)$ can be estimated as in [6]. 


\section{Numerical Example}

We apply the proposed RB method to a contracting channel problem. Define the parameter-dependent domain by $\left.\Omega^{\mu}=\left(\left(-1, \mu_{2}\right)\right) \times\left(-\mu_{1}, \mu_{1}\right)\right) \cup\left(\left(\mu_{2}, 1\right) \times\left(-\mu_{3}, \mu_{3}\right)\right)$, where $\mu \in \mathcal{D}=(0.5,0.8) \times(-0.3,0.3) \times$ $(0.2,0.3)$. We consider Dirichlet boundary conditions, except for the periodic connection between $\{-1\} \times$ $\left(-\mu_{3}, \mu_{3}\right)$ and $\{1\} \times\left(-\mu_{3}, \mu_{3}\right)$. Primal-dual formulation is used here. Finally, let $\mathbf{f} \equiv(1,0)$ and define the output of interest as $\int_{-\mu_{3}}^{\mu_{3}} \mathbf{u}_{1}(\mu)\left(\mu_{2}, y\right) \mathrm{d} y$, which is the discharge through the contraction interface. We reduced the affine decompositions to $Q_{A}=14$ and $R=4$ and ran the successive constraints method [3] to obtain a lower bound of the inf-sup constant $0<\beta_{\mathrm{SCM}}(\mu) \leq \beta_{\mathcal{N}}(\mu)$. We observed an exponential decay during the RB greedy algorithm, which is depicted in Figure 1 along with a geometry sketch and a sample solution.
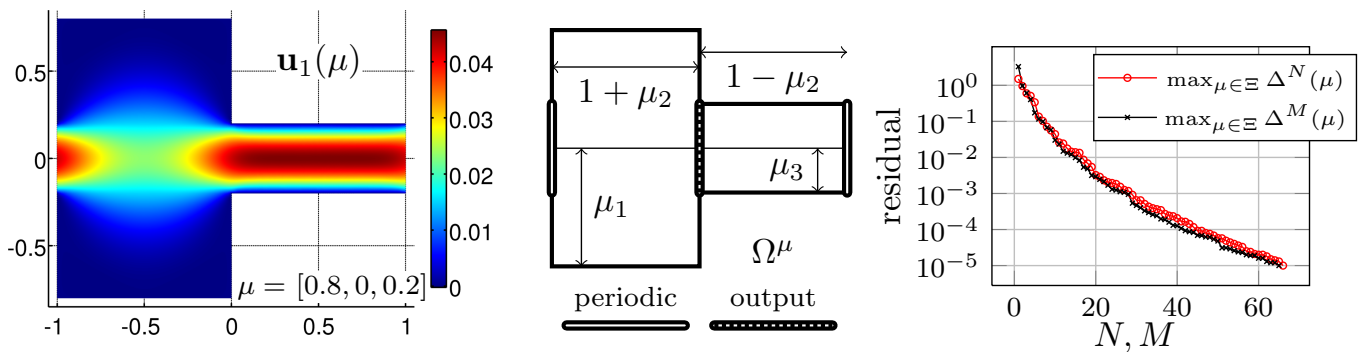

Figure 1. Numerical example from Section 4: sample plot of the horizontal velocity component (left), geometry variation (middle), and the convergence of greedy algorithm (right), where $\Xi \subset \mathcal{D}$ is the training set with 10000 points and $\Delta^{N}=\beta_{\mathrm{SCM}}(\mu)^{-1}\left\|R_{\mathrm{pr}}^{N}(\cdot ; \mu)\right\|_{\left(X^{\mathcal{N}}\right)^{\prime}}$ and $\Delta^{M}=\beta_{\mathrm{SCM}}(\mu)^{-1}\left\|R_{\mathrm{du}}^{M}(\cdot ; \mu)\right\|_{\left(X^{\mathcal{N}}\right)^{\prime}}$.

\section{References}

[1] A. Abdulle and O. Budáč, A reduced basis finite elmenet heterogeneous multiscale method for Stokes flow in porous media, in preparation, (2014).

[2] A.-L. Gerner and K. Veroy, Certified reduced basis methods for parametrized saddle point problems, SIAM Journal on Scientific Computing, 34 (2012), pp. A2812-A2836.

[3] D. B. P. Huynh, D. J. Knezevic, Y. Chen, J. S. Hesthaven, and A. T. Patera, A natural-norm successive constraint method for inf-sup lower bounds, Comput. Methods Appl. Mech. Engrg., 199 (2010), pp. 1963-1975.

[4] D. V. Rovas, Reduced-basis output bound methods for parametrized partial differential equations, PhD thesis, Massachusetts Institute of Technology, 2003.

[5] G. Rozza, D. B. P. Huynh, and A. Manzoni, Reduced basis approximation and a posteriori error estimation for Stokes flows in parametrized geometries: roles of the inf-sup stability constants, Numer. Math., 125 (2013), pp. 1-38.

[6] G. Rozza, D. B. P. Huynh, and A. T. Patera, Reduced basis approximation and a posteriori error estimation for affinely parametrized elliptic coercive partial differential equations, Arch. Comput. Methods. Eng., 15 (2008), pp. 229-275. 